\title{
Nasal Dilators (Breathe Right Strips and NoZovent) for Snoring and OSA: A Systematic Review and Meta-Analysis
}

\author{
Macario Camacho, ${ }^{1}$ Omojo O. Malu, ${ }^{2}$ Yoseph A. Kram, ${ }^{3}$ Gaurav Nigam, ${ }^{4}$ Muhammad Riaz, ${ }^{5}$ \\ Sungjin A. Song, ${ }^{3}$ Anthony M. Tolisano, ${ }^{3}$ and Clete A. Kushida ${ }^{6}$ \\ ${ }^{1}$ Tripler Army Medical Center, Division of Otolaryngology, Sleep Surgery and Sleep Medicine, 1 Jarrett White Rd, \\ Tripler AMC, Honolulu, HI 96859, USA \\ ${ }^{2}$ Uniformed Services University of the Health Sciences, 4301 Jones Bridge Road, Bethesda, MD 20814, USA \\ ${ }^{3}$ Tripler Army Medical Center, Division of Otolaryngology-Head \& Neck Surgery, Tripler AMC, Honolulu, HI 96859, USA \\ ${ }^{4}$ Clay County Hospital, 911 Stacy Burk Drive, Flora, IL 62839, USA \\ ${ }^{5}$ Sleep Disorders Center, Sunnyside Community Hospital, 1016 Tacoma Avenue, Sunnyside, WA 98944, USA \\ ${ }^{6}$ Department of Psychiatry and Behavioral Sciences, Sleep Medicine Division, Stanford Hospital and Clinics, \\ Redwood City, CA 94063, USA
}

Correspondence should be addressed to Macario Camacho; drcamachoent@yahoo.com

Received 23 July 2016; Revised 29 September 2016; Accepted 10 October 2016

Academic Editor: Dimitris Georgopoulos

Copyright ( 2016 Macario Camacho et al. This is an open access article distributed under the Creative Commons Attribution License, which permits unrestricted use, distribution, and reproduction in any medium, provided the original work is properly cited.

Objective. To systematically review the international literature for studies evaluating internal (NoZovent) and external (Breathe Right Strips) nasal dilators as treatment for obstructive sleep apnea (OSA). Study Design. Systematic review with meta-analysis. Methods. Four databases, including PubMed/MEDLINE, were searched through September 29, 2016. Results. One-hundred twelve studies were screened, fifty-eight studies were reviewed, and fourteen studies met criteria. In 147 patients, the apnea-hypopnea index (AHI) was reported, and there was an improvement from a mean \pm standard deviation $(\mathrm{M} \pm \mathrm{SD})$ of $28.7 \pm 24.0$ to $27.4 \pm 23.3 \mathrm{events} / \mathrm{hr}$, $p$ value 0.64 . There was no significant change in AHI, lowest oxygen saturation, or snoring index in OSA patients when using nasal dilators. However, a subanalysis demonstrated a slight reduction in apnea index (AI) with internal nasal dilators (decrease by 4.87 events/hr) versus minimal change for external nasal dilators (increase by 0.64 events/hr). Conclusion. Although nasal dilators have demonstrated improved nasal breathing, they have not shown improvement in obstructive sleep apnea outcomes, with the exception of mild improvement in apnea index when internal nasal dilators were used.

\section{Introduction}

The nose is composed of both internal and external structures. Although the internal structures of the nose (i.e., turbinates [1], septum [2]) do not generally move in a dynamic fashion, they can become edematous with associated symptoms of congestion and obstruction. It is known that the nose may contribute to snoring [3] and obstructive sleep apnea (OSA) when congested or obstructed. There are many treatments for OSA, to include medical management with positive airway pressure devices [4], oral appliances, and myofunctional therapy [5]. Nasal therapies to help treat OSA include nasopharyngeal airway stenting devices [6], nasal expiratory positive airway pressure devices (Provent) [7], and nasal surgery $[8,9]$.

The simple act of changing from the upright to the supine position has been shown to reduce upper airway volume by approximately $33 \%$ in OSA patients [10]. Given that the nasal cavity is upstream from the collapsible soft tissues of the upper airway, the nasal cavity directly influences the downstream airflow. Moreover, when the nasal cavity's crosssectional area increases by $10 \%$, there is a corresponding $21 \%$ increase in nasal airflow [11].

Internal (NoZovent) and external (Breathe Right Strips) nasal dilators have been studied in the treatment of OSA for over twenty years, but to date there are no published 
TABLE 1: General characteristics and quality criteria of included studies. Quality assessment of case series studies checklist from National Institute for Health and Clinical Excellence (NICE). (1) Was the case series collected in more than one center, that is, multicenter study? (2) Is the hypothesis/aim/objective of the study clearly described? (3) Are the inclusion and exclusion criteria (case definition) clearly reported? (4) Is there a clear definition of the outcomes reported? (5) Were data collected prospectively? (6) Is there an explicit statement that patients were recruited consecutively? (7) Are the main findings of the study clearly described? (8) Are outcomes stratified (e.g., by abnormal results, disease stage, and patient characteristics)?

\begin{tabular}{|c|c|c|c|c|c|c|c|c|}
\hline & $(1)$ & $(2)$ & $(3)$ & $(4)$ & $(5)$ & $(6)$ & $(7)$ & $(8)$ \\
\hline Amaro et al., 2012 & No & Yes & Yes & Yes & Yes & No & Yes & No \\
\hline Bahammam et al., 1999 & No & Yes & Yes & Yes & Yes & Yes & Yes & No \\
\hline Djupesland et al., 2001 & No & Yes & No & Yes & Yes & No & Yes & Yes \\
\hline Gosepath et al., 1999 & No & Yes & No & Yes & No & No & Yes & No \\
\hline Hoffstein et al., 1993 & No & Yes & No & Yes & Yes & No & Yes & No \\
\hline Hoijer et al., 1992 & No & Yes & Yes & Yes & Yes & Yes & Yes & Yes \\
\hline Kerr et al., 1992 & No & Yes & No & Yes & Yes & No & Yes & No \\
\hline Liistro et al., 1998 & No & Yes & No & Yes & Yes & No & Yes & Yes \\
\hline Metes et al., 1992 & No & Yes & No & Yes & Yes & Yes & Yes & No \\
\hline Pevernagie et al., 2000 & No & Yes & Yes & Yes & Yes & No & Yes & No \\
\hline Redline et al. 1998 & No & Yes & Yes & Yes & Yes & No & Yes & Yes \\
\hline Schonhofer et al., 2000 & No & Yes & Yes & Yes & Yes & Yes & Yes & No \\
\hline Todorova et al., 1998 & No & Yes & Yes & Yes & Yes & No & Yes & Yes \\
\hline Wenzel et al., 1997 & No & Yes & Yes & No & No & Yes & Yes & No \\
\hline
\end{tabular}

meta-analyses of these interventions. Therefore, the objective of this study was to systematically review the international literature, without regard to language, for studies evaluating the pretreatment and treatment data of both internal and external nasal dilators as treatment for OSA and to then use the data to perform a meta-analysis.

\section{Methods}

Three authors (M. C., O. O., and M. R.) independently searched PubMed/MEDLINE, Scopus, Embase, Google Scholar, and The Cochrane Library for studies, through September 29, 2016.

2.1. Search Strategy. Specific phrases, keywords, and MeSH terms were tailored to each database as appropriate. An example of a search strategy used in PubMed/MEDLINE includes ((("instrumentation" [Subheading]) AND ("Nasal Obstruction" [Mesh])) AND "Sleep Apnea Syndromes”) OR (("Dilatation" [Mesh] AND ("instrumentation" [Subheading])) AND ((("Sleep Apnea, Obstructive" [Mesh]) OR ("Snoring" [Mesh]) AND "Dilatation" [Mesh])) AND ("Nasal Obstruction" [Mesh])) OR ((nasal dilator [tiab]) AND ((sleep apnea [All Fields] OR (sleep apnoea [All Fields]))) OR (nasal dilatation* AND sleep apnea*) OR (nasal dilatation* AND snoring $\left.{ }^{*}\right)$ ).

2.2. Study Selection. The inclusion criteria were as follows: (1) patients: adults $\geq 18$ years old who have OSA, (2) intervention: nasal dilators, (3) comparison: sleep study data pretreatment and treatment, (4) outcome: sleep study parameters including apnea-hypopnea index (AHI), apnea index (AI), oxygen saturations, and sleepiness, and (5) study designs: all designs and all languages.
Studies reporting only qualitative outcomes and those containing a diagnosis of central sleep apnea were excluded.

2.3. Data Abstraction. Articles were independently reviewed and logged by two authors (M. C. and Y. A. K.). Patient sleep study data (i.e., lowest oxygen saturation (LSAT), mean oxygen saturation (MSAT), AHI, AI, respiratory disturbance index (RDI)), ages, body mass index (BMI), and quantitative sleepiness data (e.g., Epworth sleepiness scale (ESS) [12]) were collected. The National Institute for Health and Clinical Excellence (NICE) quality assessment tool [13] was utilized to evaluate each study; see Table 1.

2.4. Statistical Analysis. Our null hypothesis is that no difference exists in the outcomes when comparing pretreatment and treatment data. Statistical calculations were performed with STATA 14.1 (StataCorp, College Station, Texas, USA) and Review Manager Software (REVMAN) version 5.3 (Copenhagen: The Nordic Cochrane Centre: The Cochrane Collaboration, 2014). Means (M) and standard deviations (SD) were calculated with STATA 14.1. Mean differences (MD), standardized mean differences (SMD), SD, and 95\% confidence intervals [95\% CI] were calculated with the REVMAN program. Statistical significance was defined as a $p$ value $<0.05$. Random effects modeling was calculated. Cohen's guidelines [14] were followed when assigning either a small, medium, or large magnitude of effect. If a study reported the means but did not report the standard deviation, then the weighted average from the other studies in the meta-analysis was utilized (consistent with a technique from a previously published meta-analysis) [15].

Heterogeneity was evaluated using the Cochrane $Q$ statistic ( $Q$-statistic), with significance defined as a $p$ value $\leq 0.10$ [16]. The $I^{2}$ was used to evaluate for inconsistency [17]. 


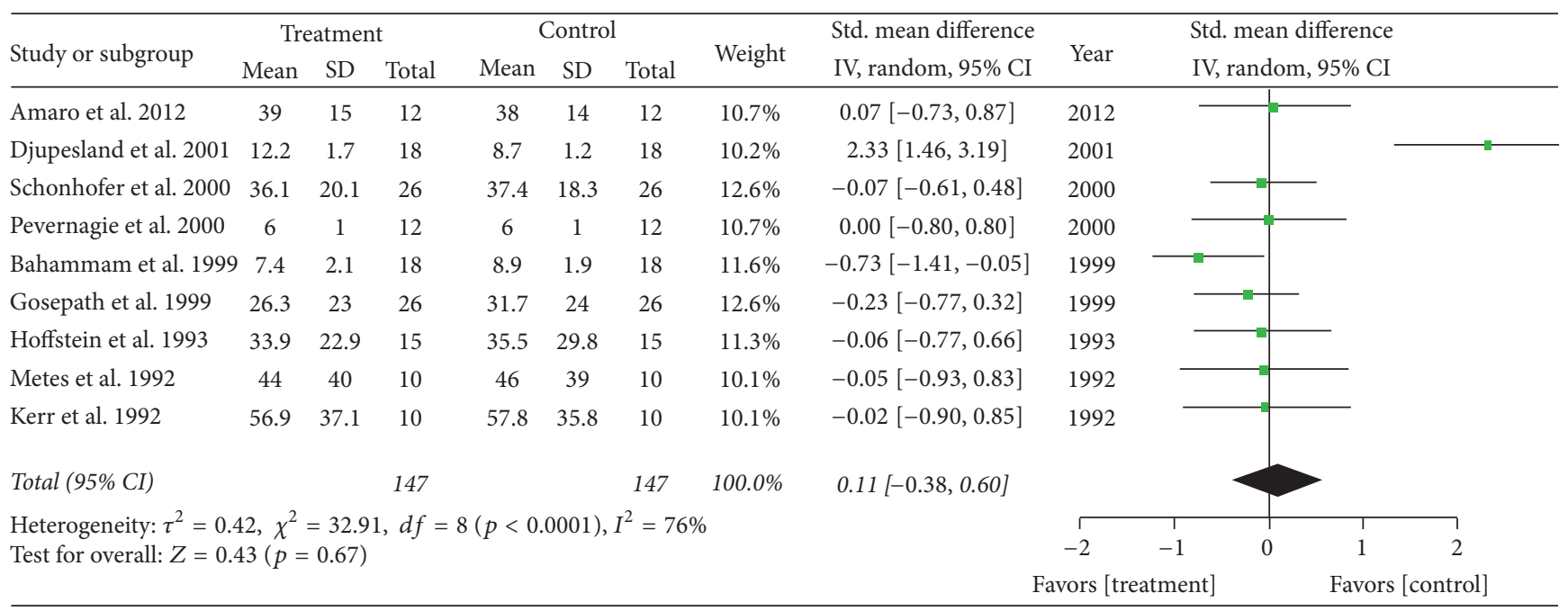

Figure 1: Standardized mean difference for apnea-hypopnea index. The overall standardized mean difference is $0.11[-0.38,0.60]$ corresponding to a small effect.

\section{Results}

One-hundred twelve studies were potentially relevant, fiftyeight studies were downloaded in full text form, twentyfour studies had data, and fourteen studies [18-31] had quantitative polysomnography data; see Table 2. Internal nasal dilators were used in five studies and external nasal dilator strips were used in nine studies.

3.1. Polysomnography Scoring Criteria. There was significant heterogeneity in polysomnographic scoring criteria among studies. Specifically, Amaro et al. defined a hypopnea as a drop of $>50 \%$ in the scoring signals with $\geq 3 \%$ oxygen desaturation. Bahammam et al. defined a hypopnea as $\geq 3 \%$ oxygen desaturation for 6 seconds or more, whereas Hoijer et al. used a $\geq 4 \%$ oxygen desaturation. Pevernagie et al. scored hypopneas when there was a $\geq 2 \%$ oxygen desaturation for at least 10 seconds. Finally, Redline et al. defined hypopneas as a discernable change in airflow lasting at least 10 seconds corresponding to a $2.5 \%$ decrease in $\mathrm{O}_{2}$ saturation or resulting in arousal. The remaining studies either stated that they followed standard polysomnography scoring criteria or did not mention the scoring criteria used.

3.2. The Effect of Nasal Dilators on Sleep Stages. The total percentage of total sleep time (TST) spent in sleep stages N1, $\mathrm{N} 2$, and N3 and rapid eye movement (REM) were evaluated. The respective pretreatment and treatment values for percent TST spent in each stage were $\mathrm{N} 1 / \mathrm{N} 2=62.8 \%$ and $63.8 \%$; $\mathrm{N} 3=20.2 \pm 14.6 \%$ and $17.6 \pm 15.6 \%$ (two-tailed $p$ value $=$ 0.2183 ); $\mathrm{REM}=17.0 \pm 7.3 \%$ and $18.6 \pm 6.1 \%$ (two-tailed $p$ value $=0.0894)$. Overall, there was no statistically significant or clinically significant difference in the sleep architecture.

3.3. Apnea-Hypopnea Index and Respiratory Disturbance Index. Ten studies reported AHI outcomes and nine provided $\mathrm{M} \pm \mathrm{SD}$. There were a total of 147 patients who had a combined pretreatment and treatment $\mathrm{M} \pm \mathrm{SD}$ of $28.7 \pm 24.0$ and $27.4 \pm 23.3$ events/hr, $p$ value 0.64 ; see Table 2 . Random effects modeling demonstrated a MD of 0.36 events/hr [95\% CI $-2.05,2.77]$ and overall effect $z=0.3$ ( $p$ value $=0.77)$. There was significant heterogeneity with a $Q$-statistic $p$ value $<0.00001$, and high inconsistency with an $I^{2}=83 \%$. The SMD was 0.11 [ $95 \% \mathrm{CI}-0.38,0.60$ ], overall effect $z=0.43$ ( $p$ value 0.67), demonstrating a minimal to small effect using Cohen's guidelines (see Table 3). There was significant heterogeneity (Q-statistic $p$ value $<0.0001)$ and high inconsistency with an $I^{2}=76 \%$, see Figure 1 . A sensitivity analysis for both MD and SMD demonstrated that after the removal of the study by Djupesland et al., there was no heterogeneity and no inconsistency, with an $I^{2}=0 \%$. The respiratory disturbance index was reported by one study (Redline et al.) with 46 patients having a pretreatment RDI of $11.8 \pm 9.6$ events $/ \mathrm{hr}$ and a treatment RDI of $9.8 \pm 9.3$ events/hr.

3.4. Apnea Index. A total of eleven studies reported AI outcomes. Ten studies comprising 190 patients had pretreatment and treatment data, with an $\mathrm{AI} \mathrm{M} \pm \mathrm{SD}$ of $23.4 \pm 25.7$ and $21.0 \pm 20.3$ events $/ \mathrm{hr}, p$ value $=0.31$, see Table 2 . Random effects modeling demonstrated a MD of -0.01 events/hr [95\% CI $-2.01,1.99]$, overall effect $z=0.01(p$ value $=0.99)$. There was no statistically significant heterogeneity ( $Q$-statistic 0.34) and minimal to low inconsistency, with an $I^{2}$ $=12 \%$. The SMD was -0.06 [95\% CI $-0.28,0.15]$, overall effect $z=0.58$ ( $p$ value 0.56 ), see Table 3 . There was no statistically significant heterogeneity $(Q$-statistic $p$ value $=$ $0.39)$ and minimal inconsistency $\left(I^{2}=7 \%\right)$. The funnel plot was clustered near the zero values, suggesting a risk of bias.

3.5. Lowest Oxygen Saturation. Ten studies with 208 patients reported LSAT outcomes and had pretreatment and treatment data for LSAT, with a $\mathrm{M} \pm \mathrm{SD}$ of $78.7 \pm 13.4$ and $79.1 \pm 12.4 \%$, $p$ value $=0.75$, see Table 2 . Random effects 


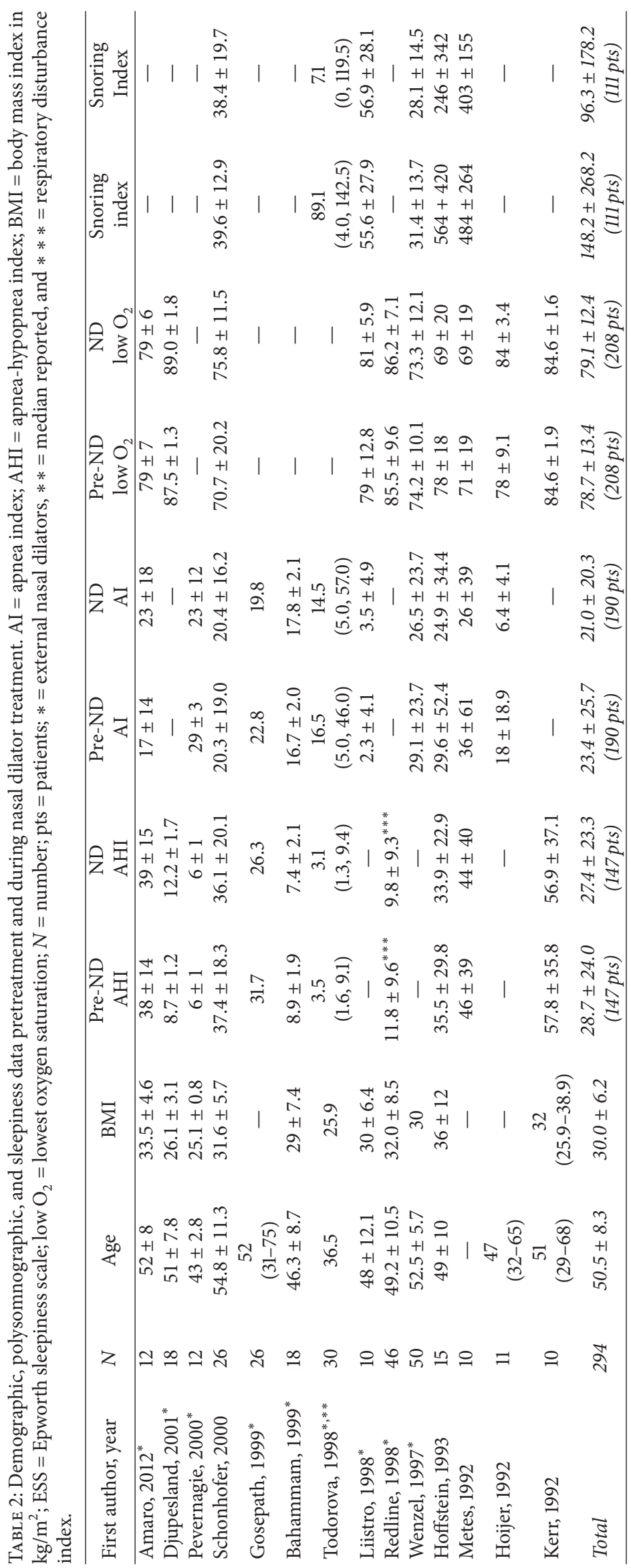


TABLE 3: Summary for mean differences and standardized mean differences. $\mathrm{AHI}=$ apnea-hypopnea index, $\mathrm{AI}=$ apnea index, $\mathrm{CI}=\mathrm{confidence}$ interval, LSAT = lowest oxygen saturation, $\mathrm{MD}=$ mean difference, $\mathrm{SI}=$ snoring index, and $\mathrm{SMD}=$ standardized mean difference.

\begin{tabular}{lccc}
\hline \multicolumn{5}{c}{$\begin{array}{c}\text { Control and nasal dilator } \\
\text { Treatment Data }\end{array}$} & \\
\hline Random effects modeling MD & MD [95\% CI $]$ & Overall effect $z$ & 0.3 \\
AHI & $0.36[-2.05,2.77]$ & 0.37 & $\mathbf{0 . 7 7}$ \\
AI & $-0.01[-2.01,1.99]$ & 0.01 & $\mathbf{0 . 9 9}$ \\
LSAT & $0.94 \%[-0.21,2.09]$ & 0.30 & $\mathbf{0 . 7 7}$ \\
SI & $-2.50[-10.7,5.71]$ & 0.60 & $\mathbf{0 . 5 5}$ \\
\hline Random effects modeling SMD & SMD [95\% CI $]$ & Cohen's magnitude of effect & \\
\hline AHI & $0.11[-0.38,0.60]$ & Small \\
AI & $-0.06[-0.28,0.15]$ & Small & \\
LSAT & $0.11[-0.38,0.60]$ & Small & \\
SI & $-0.25[-0.52,0.01]$ & Small & \\
\hline
\end{tabular}

modeling demonstrated a MD of $0.98 \%[0.09,1.87]$, overall effect $z=2.16$ ( $p$ value $=0.03$ ). There was no statistically significant heterogeneity $(Q$-statistic $p=0.40)$, and there was no to minimal inconsistency $\left(I^{2}=4 \%\right)$. The SMD was 0.14 [95\% CI $-0.10,0.38]$, overall effect $z=1.18$ ( $p$ value $=$ $0.24)$, see Table 3 . There was no significant heterogeneity $(Q-$ statistic $p$ value $=0.20$ ) and low to moderate inconsistency $\left(I^{2}=26 \%\right)$. The funnel plot is clustered toward the center, suggesting a risk of publication bias.

3.6. Scoring Criteria for Snoring. Hoijer et al. [22] measured snoring by the number of epochs with sound energy level above $55 \mathrm{~dB}$ and found a significant reduction in snoring. Liistro et al. [29] found no difference in snoring index with nasal dilators, but defined snoring index as the number of 30 second sleep epochs with at least one snore over the total number of sleep epochs. Metes et al. did not report methodology for snoring index calculation. Pevernagie et al. [31] calculated the ratio of the sum of all the individual snores with peak level of at least $2 \mathrm{~dB}$ above background noise and total sleep time and showed a significant improvement in frequency but not loudness with nasal dilators. Schonhofer et al. [26] calculated the snoring index from the number of intervals between two snores that were greater than 11 seconds but less than 60 seconds in duration, and found no objective improvement but that the majority of bed partners reported a mild reduction in snoring when their spouse used the nasal dilator. Todorova et al. [27] obtained multiple snoring variables to include snoring vibrations (maximum and mean), noise index and snoring index. They calculated snoring index as the number of snores with snoring intensity level greater than $10 \mathrm{~dB}, 15 \mathrm{~dB}, 20 \mathrm{~dB}, 25 \mathrm{~dB}$, and $30 \mathrm{~dB}$ per hour. They found only a significant decrease in three subcategories in all the 34 snoring variables observed, which could be explained by random error rather than a true significant finding. Wenzel et al. [28] found a significant improvement in snoring index by calculating the number of snoring events per hour.

3.7. Outcomes for Snoring. Polysomnography-based snoring index was reported by six studies. Five studies had a combined total of 111 patients provided means and standard deviations. The combined pretreatment and treatment data, and the snoring $\mathrm{M} \pm \mathrm{SD}$ were $148.2 \pm 268.2$ and $96.3 \pm$ 178.2 snores $/ \mathrm{hr}$ ( $p$ value $=0.09$ ), see Table 2 . Random effects modeling demonstrated a mean difference of $-2.5[-10.7$, 5.71], overall effect $z=0.60$ ( $p$ value 0.55 ). There was no statistically significant heterogeneity $(Q$-statistic $p$ value $=$ 0.2 ), but there was a low level of inconsistency with a value of $33 \%$. The SMD was -0.25 [95\% CI $-0.52,0.01]$, overall effect $z=1.86(p$ value $=0.06)$, with no statistically significant heterogeneity ( $Q$-statistic $p$ value 0.55$)$ and no inconsistency $\left(I^{2}=0 \%\right)$.

3.8. Sleepiness. Amaro et al., Redline et al. and Schonhofer et al. utilized the ESS for 85 patients and had a combined control and treatment $\mathrm{M} \pm \mathrm{SD}$ of $10.3 \pm 5.2$ and $9.5 \pm 5.6$, respectively $(p$ value $=0.34)$.

3.9. Subanalysis: Internal vs External Nasal Dilators. Subanalysis showed that the external nasal dilators increased the AHI in 86 patients by $0.48[-2.13,3.09]$ events $/ \mathrm{hr}$, overall effect $z=$ 0.36 ( $p$ value 0.72 ), while the internal nasal dilators reduced the AHI by $1.37[-9.91,7.16]$ events $/ \mathrm{hr}$, overall effect $z=0.32$ ( $p$ value 0.75 ). Subanalysis of apnea index showed that the external nasal dilators increased the $\mathrm{AI}$ in 128 patients by 0.64 $[-0.98,2.27]$ events/hr, overall effect $z=0.78$ ( $p$ value 0.44 ), while internal nasal dilators changed the $\mathrm{AI}$ in 62 patients by $-4.87[-11.94,2.20]$ events/hr, overall effect $z=1.35$ ( $p$ value $=0.18$ ). Subanalysis of lowest oxygen saturation showed that the external nasal dilators improved the LSAT in 136 patients by $1.29 \%[0.35,2.23]$, overall effect $z=2.69$ ( $p$ value $=0.007$ ), while the internal nasal dilators improved the LSAT in 72 patients by $1.47 \%[-2.50,5.43]$, overall effect $z=0.72(p$ value $=0.47)$. Subanalysis of snoring outcomes showed that the external nasal dilators improved snoring in 60 patients by $-3.08[-8.47,2.32]$ snores $/ \mathrm{hr}$, overall effect $z=1.12$ ( $p$ value $=0.26$, while internal nasal dilators improved snoring in 51 patients by $-86.54[-241.0,67.9]$ snores/hr, overall effect $z=1.10(p$ value $=0.27)$.

3.10. The Effect of Disease Duration, Smoking, Alcohol, and $B M I$. No study reported the disease duration. Regarding 
BMI, Amaro et al., Liistro et al., Redline et al., and Schonhofer et al. did not stratify outcomes based on the BMI; however, they did report that the BMI values did not change during the intervention period. Regarding alcohol and tobacco, (1) Bahammam et al. instructed patients to avoid alcohol during the study, (2) Redline et al. described instructing patients to have good sleep hygiene to include no tobacco or alcohol near bedtime, (3) Schonhofer et al. instructed patients to abstain from alcohol for 1 week prior to both the baseline polysomnographic recordings and the follow-up study, and (4) Todorova et al. reported that all subjects were free of alcohol during the study.

\section{Discussion}

Although nasal dilators may subjectively improve a patient's nasal obstruction, the devices have not been demonstrated improving sleep study parameters. Therefore, the devices should not be thought of as curative for OSA but rather should be considered as adjuncts to treatment. For example, nasal dilators may reduce the pressure required for continuous positive airway pressure (CPAP) devices [32]. Since lower CPAP treatment pressures often improve CPAP use [8], it is possible that nasal dilator use may also improve CPAP use. In those patients with significant improvement in breathing with nasal dilators but who cannot tolerate the devices, site directed nasal surgery may be considered. The therapeutic implications for patients who can tolerate and do receive benefit, long-term use may be considered.

Twelve of the fourteen studies in this review showed no significant change in AHI with the use of nasal dilators $[18,19,21,23-31]$. There was a slight reduction in AI with internal nasal dilators $(-4.87$ events/hr). Notably, the Djupesland et al. [19] study actually demonstrated a significant increase in AHI with nasal dilator compared to placebo. Only two studies (both using internal nasal dilators), Gosepath et al. [20] and Hoijer et al. [22], demonstrated a significant $17 \%$ reduction in AHI from 31.7 to 26.3 events/hr. In the studies that showed an improvement in obstructive events the patients were not cured of OSA. Additionally, studies did not control for body position. Like the AHI, there was only a minimal change in LSAT with nasal dilator treatment. The overall mean pretreatment and treatment LSAT values improved from $76.8 \pm 13.7 \%$ to $77.1 \pm 12.8 \%$, which was not a significant improvement. These findings are consistent with the understanding that the nose generally is not considered a site of obstruction during apneic events, since the nose does not move dynamically. The more susceptible areas of collapse include the oropharynx, hypopharynx, soft palate, and epiglottis in OSA patients [21,33].

Snoring demonstrated improvement when the overall raw data was combined, but there was only a small improvement when random effects modeling was applied. A subanalysis comparing external versus internal nasal dilators demonstrated that the external nasal dilators improved the snoring index minimally (-3.08), but the internal nasal dilators improved snoring more significantly (-86.54). It is unclear why the internal nasal dilators would decrease snoring more than external nasal dilators, but a possible explanation is variability in how the snoring index was defined and measured. The diverse calculation for snoring index in the included studies could explain the mixed improvement with nasal dilators. A uniform method for measuring snoring should be used in future studies in order to facilitate interpretation among studies.

\section{Limitations}

The inherent limitation in this systematic review is the lower quality of published studies evaluating nasal dilators. Most studies included were individual case-control or prospective case series studies, often with small sample sizes, lacking randomization and having other significant drawbacks. Therefore, in order to improve the level of evidence, additional, prospective trials with randomization are needed. As with any systematic review, it is possible despite best efforts that studies were missed in searching the literature. There may be differences in polysomnogram scoring criteria among institutions; however, these differences were not specified by the articles included in this review.

\section{Conclusion}

Although nasal dilators have demonstrated improved nasal breathing, they have not shown improvement in obstructive sleep apnea outcomes, with the exception of mild improvement in apnea index when internal nasal dilators were used.

\section{Disclosure}

The views expressed in this manuscript are those of the author(s) and do not reflect the official policy or position of the Department of the Army, Department of Defense, or the US Government. Institution where the work was primarily performed is Tripler Army Medical Center, Hawaii.

\section{Competing Interests}

The authors declare that there are no competing interests.

\section{Acknowledgments}

Dr. Kushida has research and grant support from Apnex, Seven Dreamers Lab, Resmed, and Pacific Medical; he also has a patent from Philips Respironics.

\section{References}

[1] M. Camacho, S. Zaghi, V. Certal et al., "Inferior Turbinate classification system, grades 1 to 4: development and validation study," The Laryngoscope, vol. 125, no. 2, pp. 296-302, 2015.

[2] J. Teixeira, V. Certal, E. T. Chang, and M. Camacho, "Nasal septal deviations: a systematic review of classification systems," Plastic Surgery International, vol. 2016, Article ID 7089123, 8 pages, 2016.

[3] J. C. Hsia, M. Camacho, and R. Capasso, "Snoring exclusively during nasal breathing: a newly described respiratory pattern during sleep," Sleep \& Breathing, vol. 18, no. 1, pp. 159-164, 2014. 
[4] C. E. Sullivan, F. G. Issa, M. Berthon-Jones, and L. Eves, "Reversal of obstructive sleep apnoea by continuous positive airway pressure applied through the nares," The Lancet, vol. 317, no. 8225, pp. 862-865, 1981.

[5] M. Camacho, V. Certal, J. Abdullatif et al., "Myofunctional therapy to treat obstructive sleep apnea: a systematic review and meta-analysis," Sleep, vol. 38, no. 5, pp. 669-675, 2015.

[6] A. R. Kumar, C. Guilleminault, V. Certal, D. Li, R. Capasso, and M. Camacho, "Nasopharyngeal airway stenting devices for obstructive sleep apnoea: a systematic review and meta-analysis," The Journal of Laryngology and Otology, vol. 129, no. 1, pp. 2-10, 2015.

[7] M. Riaz, V. Certal, G. Nigam, J. Abdullatif, and S. Zaghi, "Nasal expiratory positive airway pressure devices (Provent) for OSA: a systematic review and meta-analysis," Sleep Disorders, vol. 2015, Article ID 734798, 15 pages, 2015.

[8] M. Camacho, M. Riaz, R. Capasso et al., "The effect of nasal surgery on continuous positive airway pressure device use and therapeutic treatment pressures: a systematic review and metaanalysis," Sleep, vol. 38, no. 2, pp. 279-286, 2015.

[9] H.-Y. Li, P.-C. Wang, Y.-P. Chen, L.-A. Lee, T.-J. Fang, and H.C. Lin, "Critical appraisal and meta-analysis of nasal surgery for obstructive sleep apnea," American Journal of Rhinology and Allergy, vol. 25, no. 1, pp. 45-49, 2011.

[10] M. Camacho, R. Capasso, and S. Schendel, "Airway changes in obstructive sleep apnoea patients associated with a supine versus an upright position examined using cone beam computed tomography," The Journal of Laryngology and Otology, vol. 128, no. 9, pp. 824-830, 2014.

[11] N. B. Powell, A. I. Zonato, E. M. Weaver et al., "Radiofrequency treatment of turbinate hypertrophy in subjects using continuous positive airway pressure: a randomized, double-blind, placebo-controlled clinical pilot trial," The Laryngoscope, vol. 111, no. 10, pp. 1783-1790, 2001.

[12] M. W. Johns, "A new method for measuring daytime sleepiness: the Epworth sleepiness scale," Sleep, vol. 14, no. 6, pp. 540-545, 1991.

[13] National Institute for Health and Clinical Excellence, Methods for Development of NICE Public Health Guidance, National Institute for Health and Clinical Excellence, London, UK, 2009.

[14] J. Cohen, Statistical Power Analysis for the Behavioral Sciences, Erlbaum, Hillsdale, NJ, USA, 2nd edition, 1988.

[15] A. W. Murphey, J. A. Kandl, S. A. Nguyen, A. C. Weber, and M. B. Gillespie, "The effect of glossectomy for obstructive sleep apnea: a systematic review and meta-analysis," Otolaryngology-Head and Neck Surgery, vol. 153, no. 3, pp. 334-342, 2015.

[16] J. Lau, J. P. A. Ioannidis, and C. H. Schmid, "Quantitative synthesis in systematic reviews," Annals of Internal Medicine, vol. 127, no. 9, pp. 820-826, 1997.

[17] J. P. T. Higgins, S. G. Thompson, J. J. Deeks, and D. G. Altman, "Measuring inconsistency in meta-analyses," British Medical Journal, vol. 327, no. 7414, pp. 557-560, 2003.

[18] A. S. Bahammam, R. Tate, J. Manfreda, and M. H. Kryger, "Upper airway resistance syndrome: effect of nasal dilation, sleep stage, and sleep position," Sleep, vol. 22, no. 5, pp. 592-598, 1999.

[19] P. G. Djupesland, O. Skatvedt, and A. K. Borgersen, "Dichotomous physiological effects of nocturnal external nasal dilation in heavy snorers: the answer to a rhinologic controversy?" American Journal of Rhinology, vol. 15, no. 2, pp. 95-103, 2001.
[20] J. Gosepath, R. G. Amedee, S. Romantschuck, and W. J. Mann, "Breathe right nasal strips and the respiratory disturbance index in sleep related breathing disorders," The American Journal of Rhinology, vol. 13, no. 5, pp. 385-389, 1999.

[21] V. Hoffstein, S. Mateika, and A. Metes, "Effect of nasal dilation on snoring and apneas during different stages of sleep," Sleep, vol. 16, no. 4, pp. 360-365, 1993.

[22] U. Hoijer, H. Ejnell, J. Hedner, B. Petruson, and L. B. Eng, "The effects of nasal dilation on snoring and obstructive sleep apnea," Archives of Otolaryngology-Head and Neck Surgery, vol. 118, no. 3, pp. 281-284, 1992.

[23] P. Kerr, T. Millar, P. Buckle, and M. Kryger, "The importance of nasal resistance in obstructive sleep apnea syndrome," The Journal of Otolaryngology, vol. 21, no. 3, pp. 189-195, 1992.

[24] A. Metes, P. Cole, V. Hoffstein, and H. Miljeteig, "Nasal airway dilation and obstructed breathing in sleep," The Laryngoscope, vol. 102, no. 9, pp. 1053-1055, 1992.

[25] S. Redline, N. Adams, M. E. Strauss, T. Roebuck, M. Winters, and C. Rosenberg, "Improvement of mild sleep-disordered breathing with CPAP compared with conservative therapy," American Journal of Respiratory and Critical Care Medicine, vol. 157, no. 3, pp. 858-865, 1998.

[26] B. Schonhofer, K. A. Franklin, H. Brunig, H. Wehde, and D. Kohler, "Effect of nasal-valve dilation on obstructive sleep apnea," Chest, vol. 118, no. 3, pp. 587-590, 2000.

[27] A. Todorova, R. Schellenberg, H. C. Hofmann, and W. Dimpfel, "Effect of the external nasal dilator Breathe Right on snoring," European Journal of Medical Research, vol. 3, no. 8, pp. 367-379, 1998.

[28] M. Wenzel, B. Schonhofer, K. Siemon, and D. Kohler, "Nasal strips without effect on obstructive sleep apnea and snoring," Pneumologie, vol. 51, no. 12, pp. 1108-1110, 1997.

[29] G. Liistro, P. Rombaux, M. Dury, T. Pieters, G. Aubert, and D. O. Rodenstein, "Effects of breathe right on snoring: A Polysomnographic Study," Respiratory Medicine, vol. 92, no. 8, pp. 10761078, 1998.

[30] A. C. S. Amaro, F. H. G. Duarte, R. S. Jallad, M. D. Bronstein, S. Redline, and G. Lorenzi-Filho, "The use of nasal dilator strips as a placebo for trials evaluating continuous positive airway pressure," Clinics, vol. 67, no. 5, pp. 469-474, 2012.

[31] D. Pevernagie, E. Hamans, P. Van Cauwenberge, and R. Pauwels, "External nasal dilation reduces snoring in chronic rhinitis patients: a randomized controlled trial," The European Respiratory Journal, vol. 15, no. 6, pp. 996-1000, 2000.

[32] B. Schönhofer, J. Kerl, S. Suchi, D. Köhler, and K. A. Franklin, "Effect of nasal valve dilation on effective CPAP level in obstructive sleep apnea," Respiratory Medicine, vol. 97, no. 9, pp. 10011005, 2003.

[33] C. Torre, M. Camacho, S. Y.-C. Liu, L.-K. Huon, and R. Capasso, "Epiglottis collapse in adult obstructive sleep apnea: a systematic review," The Laryngoscope, vol. 126, no. 2, pp. 515-523, 2016. 


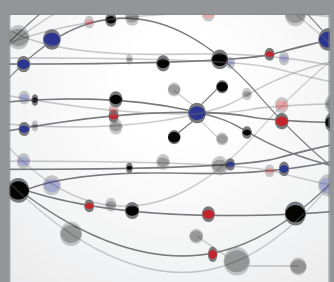

The Scientific World Journal
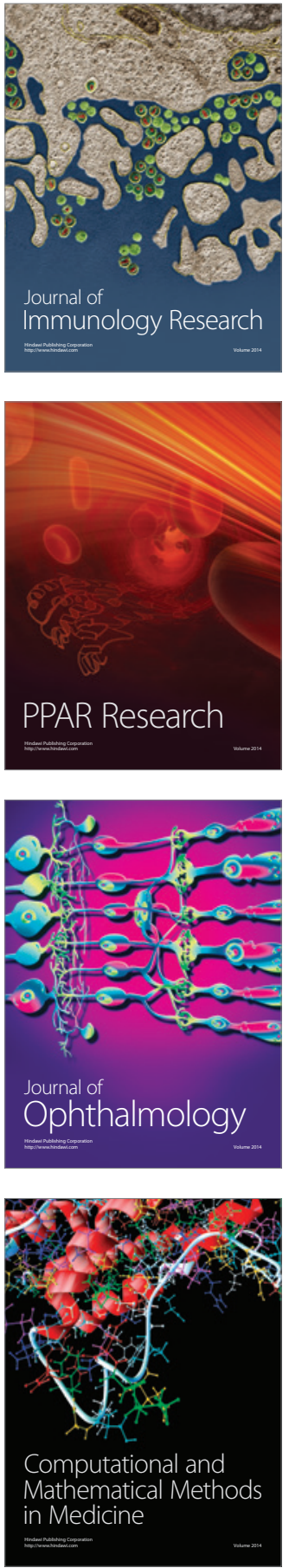

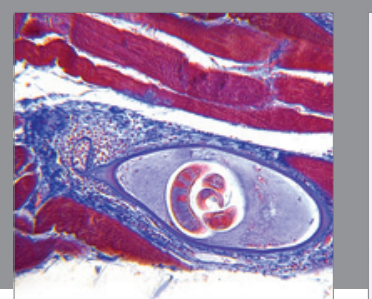

Gastroenterology Research and Practice

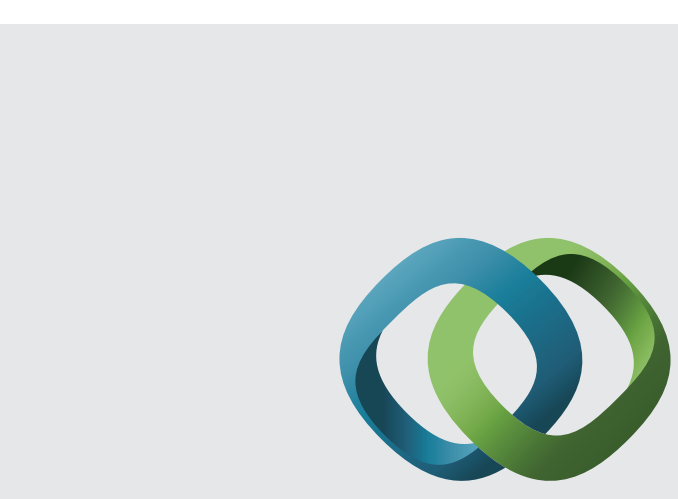

\section{Hindawi}

Submit your manuscripts at

http://www.hindawi.com
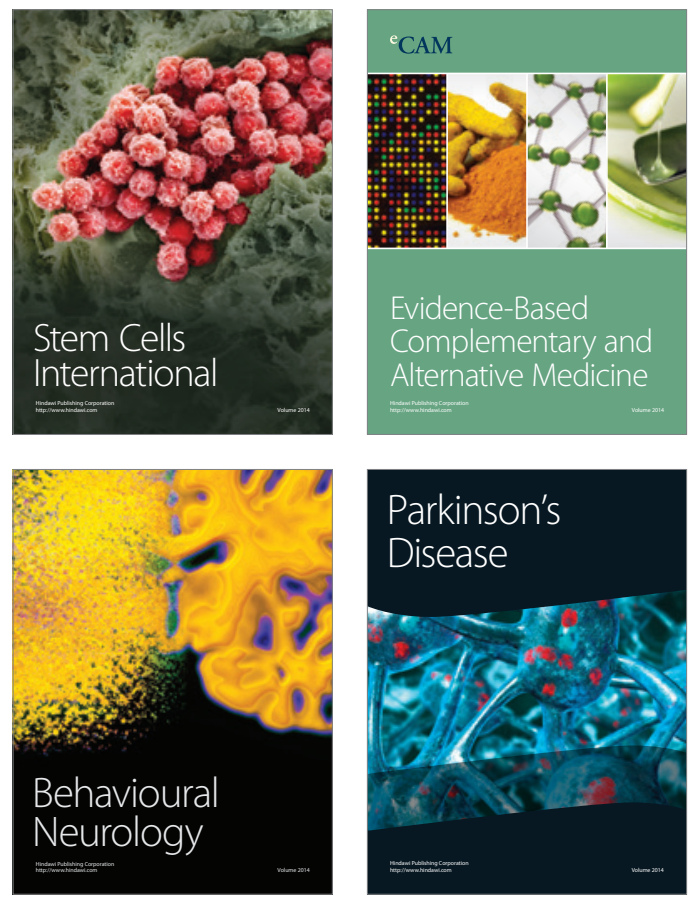
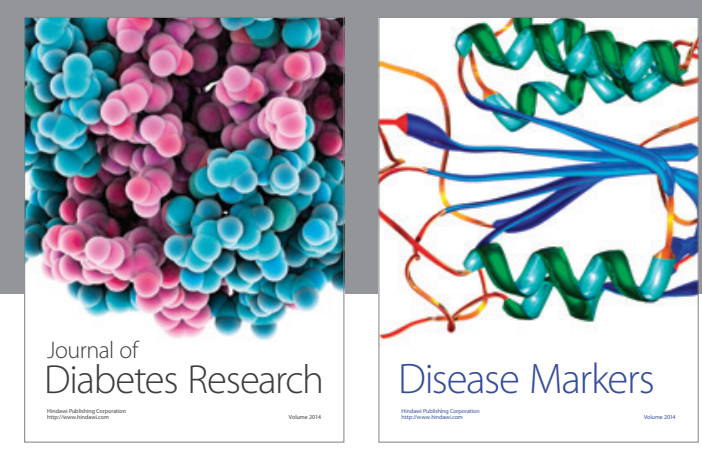

Disease Markers
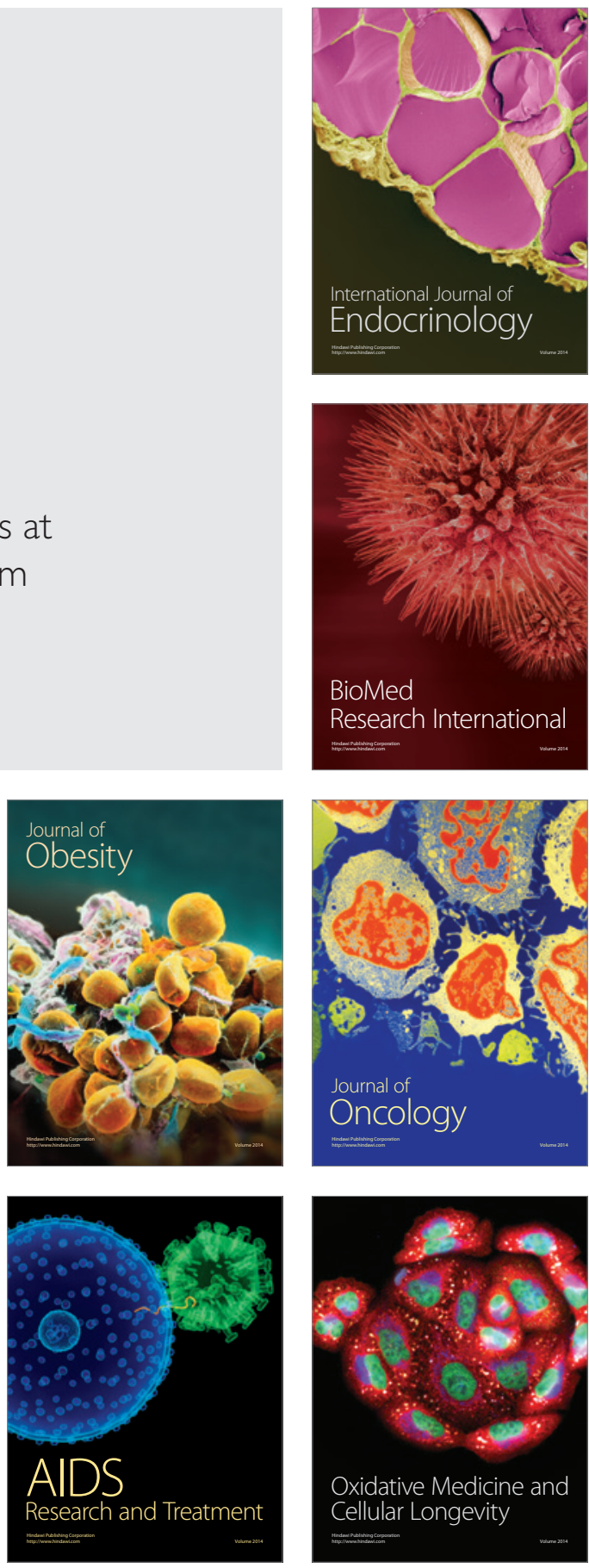